\title{
On the structure of solutions to a class of quasilinear elliptic Neumann problems. Part II
}

\author{
Chunshan Zhao and \\ Department of Mathematical Sciences, Georgia Southern University, Statesboro, GA 30460, USA \\ Yi Li \\ Department of Mathematics, University of lowa, lowa City, IA 52242-1419, USA \\ yli@math.uiowa.edu; Department of Mathematics, Xian Jiaotong University, Xian, Shaanxi, China
}

\begin{abstract}
We continue our work (Y. Li, C. Zhao in J Differ Equ 212:208-233, 2005) to study the structure of positive solutions to the equation $\varepsilon^{m} \Delta_{m} u-u^{m-1}+f(u)=0$ with homogeneous Neumann boundary condition in a smooth bounded domain of $\mathbb{R}^{N}(N \geq 2)$. First, we study subcritical case for $2<m<N$ and show that after passing by a sequence positive solutions go to a constant in $C^{1, \alpha}$ sense as $\varepsilon \rightarrow \infty$. Second, we study the critical case for $1<m<N$ and prove that there is a uniform upper bound independent of $\varepsilon \in[1, \infty)$ for the least-energy solutions. Third, we show that in the critical case for $1<m \leq 2$ the least energy solutions must be a constant if $\varepsilon$ is sufficiently large and for $2<m<N$ the least energy solutions go to a constant in $C^{l, \alpha}$ sense as $\varepsilon \rightarrow \infty$.
\end{abstract}

\section{Introduction}

Let $\Omega \subseteq \mathbb{R}^{N}(N \geq 2)$ be a smooth bounded domain. We continue our former work [8] to investigate the structure of positive solutions to a class of quasilinear elliptic Neumann problems as follows:

$$
\begin{cases}\varepsilon^{m} \Delta_{m} u-u^{m-1}+f(u)=0 & \text { in } \Omega, \\ u>0 & \text { in } \Omega, \\ \frac{\partial u}{\partial n}=0 & \text { on } \partial \Omega,\end{cases}
$$

where $\Delta_{m} u=\operatorname{div}\left(|\nabla u|^{m-2} \nabla u\right), 1<m<N$ and $n$ is the unit outer normal of $\partial \Omega$. The coefficient $\varepsilon>0$ is a parameter.

The problem (1)-(3) appears in the study of non-Newtonian fluids, chemotaxis, and biological pattern formation. For example, in the study of non-Newtonian fluids, the quantity $m$ is a characteristic of the medium. Media with $m>2$ are called dilatant fluids and those with $m<2$ are called pseudoplastics. If $m=2$, they are Newtonian fluids (see [2] and its bibliography). In this special case $m=2,(1)-(3)$ is also known as the stationary equation of the Keller-Segal system in chemotaxis (see [11]) or the limiting stationary equation of the so-called Giener-Meinhardt system in biological pattern formation [19].

(C) Springer-Verlag 2009

czhao@GeorgiaSouthern.edu . 
For the case $m=2$, the asymptotic behavior of mountain-pass solutions to the system (1)-(3) as $\varepsilon \rightarrow 0^{+}$was studied by Lin, Ni, and Takagi in a series of remarkable papers $[11,13,14]$. First, Lin et al. [11] applied the mountain-pass lemma [1] to show the existence of a mountain-pass solution $u_{\varepsilon}$ to (1)-(3). Under the assumption that $\frac{f(t)}{t}$ is increasing on $\mathbb{R}^{+}$it was shown [14] that every mountain-pass solution is a least-energy solution, by which it is meant that $u_{\varepsilon}$ has the least energy among all the solutions to (1)-(3), with the energy functional defined by

$$
I_{\varepsilon}(u)=\int_{\Omega}\left(\frac{\varepsilon^{2}}{2}|\nabla u|^{2}+\frac{1}{2} u^{2}-F\left(u_{+}\right)\right) d x,
$$

where $u_{+}=\max \{u, 0\}$ and $F\left(u_{+}\right)=\int_{0}^{u_{+}} f(s) d s$. They also gave some important preliminary results about the mountain-pass solutions. In [13,14], Ni and Takagi investigated the asymptotic behavior of the least-energy solution $u_{\varepsilon}$ as $\varepsilon \rightarrow 0^{+}$and showed that the leastenergy solution has a single-spike which is achieved at a point on $\partial \Omega$ and moreover for $\varepsilon>0$ sufficiently small this point stays at a point where maximum of mean curvature of $\partial \Omega$ is attained. For the general case $1<m<N$, it was shown $[9,10]$ that as $\varepsilon \rightarrow 0^{+}$the global maximum point of the least-energy solution $u_{\varepsilon}$ approaches a point where maximum of mean curvature of $\partial \Omega$ is attained at a rate of $o(\varepsilon)$. Also see [3-7] for existence and properties of multiple-peaks solutions for the case $m=2$.

In our former work [8] we gave some a-priori estimates of mountain-pass solutions and investigated uniform upper bound and Harnack inequalities etc for all positive solutions of (1)-(3) under assumption $1<m<N$ and the following assumptions on $f$.

$$
\begin{aligned}
& \left(\mathrm{f}_{1}\right): f: \mathbb{R} \rightarrow \mathbb{R} \text { is a continuous function and } f:(0, \infty) \rightarrow(0, \infty) \text { is } C^{1} . \\
& \left(\mathrm{f}_{2}\right): f(t) \equiv 0 \text { for } t \leq 0 \text { and } \frac{f(t)}{t^{m-1}} \rightarrow 0 \text { as } t \rightarrow 0^{+} \text {and } f(t)>0 \text { for } t>0 . \\
& \left(\mathrm{f}_{3}\right): \lim _{t \rightarrow \infty} \frac{f(t)}{t^{p}}=B>0 \text { with } m-1<p<v-1 \text { and } v=\frac{N m}{N-m} \text {. } \\
& \left(\mathrm{f}_{4}\right): \text { Let } F(t)=\int_{0}^{t} f(s) d s d s \text {. There exists a constant } \theta>m \text { such that } 0 \leq \theta F(t) \leq t f(t) \\
& \text { for } t \geq 0 \text {. }
\end{aligned}
$$

Especially we proved the following results (see $[11,12]$ for the case $m=2$ ).

\section{Theorem A}

([8], Theorem 1.3, P. 210) Under assumptions $\left(\mathrm{f}_{1}\right)-\left(\mathrm{f}_{4}\right)$, for any positive solution $\mathrm{u}_{\varepsilon} \in \mathrm{W}^{1, \mathrm{~m}}$ $(\Omega)$ to (1)-(3), there exists a constant $\mathrm{C}$ independent of $\varepsilon \in(0,+\infty)$ such that

$$
\sup _{x \in \Omega} u_{\varepsilon}(x) \leq C .
$$

Moreover if $1<\mathrm{m} \leq 2$, there exists a positive constant $\varepsilon_{1}$ sufficiently large such that for $\varepsilon>$ $\varepsilon_{1}$, any positive solution to the problem (1)-(3) that belongs to $\mathrm{W}^{1, \mathrm{~m}}(\Omega)$ must be a constant.

In this paper we continue to investigate asymptotic behaviors of all solutions of (1)-(3) under assumptions of $\left(\mathrm{f}_{1}\right)-\left(\mathrm{f}_{4}\right)$ with $2<m<N$, and least-energy solutions of (1)-(3) with $1<$ 
$m<N$ and $f(u)=u_{+}^{\frac{N(m-1)+m}{N-m}}$ (i.e., the critical case) as $\varepsilon \rightarrow \infty$. For $1<m<N$ and $f(u)=u_{+}^{\frac{N(m-1)+m}{N-m}}$ associated with (1)-(3) is the functional $J_{\varepsilon}: W^{1, m}(\Omega) \rightarrow \mathbb{R}$ defined by

$$
J_{\varepsilon}(v)=\frac{1}{m} \int_{\Omega}\left(\varepsilon^{m}|\nabla v|^{m}+|v|^{m}\right) d x-\frac{N-m}{N m} \int_{\Omega} v_{+}^{\frac{N m}{N-m}} d x .
$$

Let

$$
\Gamma_{\text {set }}=\left\{h \in C\left([0,1] ; W^{1, m}(\Omega)\right) \mid h(0)=0, h(1)=e\right\},
$$

where $e \not \equiv 0$ is a nonnegative function in $W^{1, m}(\Omega)$ with $J_{\varepsilon}(e) \leq 0$ (e.g., a sufficiently large constant). Later we will see

$$
c_{\varepsilon}=\inf _{h \in \Gamma_{\mathrm{set}} 0 \leq t \leq 1} \max _{\varepsilon}(h(t))
$$

is a positive critical value of $J_{\varepsilon}$.

Moreover, since $t^{\frac{N(m-1)+m}{N-m}} / t^{m-1}=t^{\frac{m^{2}}{N-m}}$ is increasing for $t>0$ it is known [9] that $c_{\varepsilon}$ can also characterized as

$$
c_{\varepsilon}=\inf \left\{M_{*}[v] \mid v \in W^{1, m}(\Omega), v \not \equiv 0 \text { and } v \geq 0 \text { in } \Omega\right\}
$$

with

$$
M_{*}[v]=\sup _{t \geq 0} J_{\varepsilon}(t v)
$$

Hence $c_{\varepsilon}$ is the least among all positive critical values of $J_{\varepsilon}$. Therefore we call such a critical point $u_{\varepsilon}$ of $J_{\varepsilon}$ with $J_{\varepsilon}\left(u_{\varepsilon}\right)=c_{\varepsilon}$ a least-energy solution. Now our results can be stated as follows:

\section{Theorem 1}

Under assumptions $2<\mathrm{m}<\mathrm{N}$ and $\left(\mathrm{f}_{1}\right)-\left(\mathrm{f}_{4}\right)$ on $\mathrm{f}$, for any positive solution $\mathrm{u}_{\varepsilon} \in \mathrm{W}^{1, \mathrm{~m}}$ with $\varepsilon$ $\in[1, \infty)$ there exist two positive constants $\mathrm{C}$ independent of $\varepsilon$ or $\mathrm{u}_{\varepsilon}$ and $\alpha=\alpha(\mathrm{m}, \mathrm{N}, \Omega) \in$ $(0,1)$ such that

$$
\left\|u_{\varepsilon}-\bar{u}_{\varepsilon}\right\|_{C^{1, \alpha}(\Omega)} \leq C \varepsilon^{-\beta},
$$

where $\beta=\min \left\{\frac{m^{2}}{N(m-2)}, \frac{m}{m-1}\right\}$ and $\bar{u}_{\varepsilon}$ is a positive constant defined as the average of $\mathrm{u}_{\varepsilon}$ over, $\Omega$ i.e., $\bar{u}_{\varepsilon}=\frac{1}{|\Omega|} \int_{\Omega} u_{\varepsilon} d x$. Especially we have $\left\|u_{\varepsilon}-\bar{u}_{\varepsilon}\right\|_{C^{1, \alpha}(-\bar{\Omega})} \rightarrow 0$ as $\varepsilon \rightarrow \infty$. Moreover, after passing by a sequence $\bar{u}_{\varepsilon}$ converges to a positive zero of $\mathrm{t}^{\mathrm{m}-1}-\mathrm{f}(\mathrm{t})$ as $\varepsilon \rightarrow \infty$. 
Next is our result about uniform upper bound of the least-energy solution $u_{\varepsilon}$ for $\varepsilon \in[1, \infty)$, $1<m<N$ and $f(u)=u_{+}^{\frac{N(m-1)+m}{N-m}}$.

\section{Theorem 2}

Suppose $1<\mathrm{m}<\mathrm{N}$ and $\bar{u}_{\varepsilon}$. For $\varepsilon \in[1, \infty)$ there exists a constant $\mathrm{C}>0$ independent of $\varepsilon$ such that any least-energy solution $\mathrm{u}_{\varepsilon}$ to (1)-(3) satisfies

$$
\sup _{x \in \Omega} u_{\varepsilon}(x) \leq C .
$$

For asymptotic behaviors of the least-energy solutions $u_{\varepsilon}$ as $\varepsilon \rightarrow \infty$, we have

\section{Theorem 3}

Let $1<\mathrm{m}<\mathrm{N}, f(u)=u_{+}^{\frac{N(m-1)+m}{N-m}}$ and $\mathrm{u}_{\varepsilon}$ be a least energy solution. Then we have

$\left(\mathrm{f}_{1}\right)$ : For $1<\mathrm{m} \leq 2$ there exists a positive constant $\varepsilon_{1}$ sufficiently large such that for $\varepsilon>$ $\varepsilon_{1}, \mathrm{u}_{\varepsilon} \equiv 1$.

$$
\left(\mathrm{f}_{2}\right) \text { : For } 2<\mathrm{m}<\mathrm{N}, \quad\left\|u_{\varepsilon}-1\right\|_{C^{1, \alpha}(\bar{\Omega})} \rightarrow 0 \text { as } \varepsilon \rightarrow \infty \text {, where } \alpha \text { is stated as in Theorem } 1 .
$$

The organization of this paper is as follows. Some a-priori estimates and proof of Theorem 1 will be presented in Sect. 2. In Sect. 3 we will discuss existence of least energy solutions and prove Theorem 2. The proof of Theorem 3 will be given in Sect. 4 .

\section{Some a-priori estimates and proof of Theorem 1}

In this section we will give some a-priori estimates related to $u_{\varepsilon}$ and use them to prove Theorem 1 . From now on $C, C_{i}(i=0,1, \ldots)$ are generic positive constants, we will specify them whenever it is necessary.

Let $u_{\varepsilon} \in W^{1, m}(\Omega)$ be a positive solution to (1)-(3) with $2<m<N$ and assumptions ( $\left.\mathrm{f}_{1}\right)-\left(\mathrm{f}_{4}\right)$ on $f$. Then we know $u_{\varepsilon} \in C^{1}(\bar{\Omega})$ and $u_{\varepsilon}$ is uniformly bounded due to Theorem A, i.e.,

$$
\sup _{x \in \Omega} u_{\varepsilon}(x) \leq C .
$$

Decompose $u_{\varepsilon}$ as $u_{\varepsilon}=\bar{u}_{\varepsilon}+v_{\varepsilon}$, where

$$
\bar{u}_{\varepsilon}=\frac{1}{|\Omega|} \int_{\Omega} u_{\varepsilon} d x \quad \text { and } \quad \int_{\Omega} v_{\varepsilon} d x=0
$$

Then it follows from (1)-(3) that $v_{\varepsilon}$ satisfies the following equations

$$
\begin{cases}\varepsilon^{m} \Delta_{m} v_{\varepsilon}-u_{\varepsilon}^{m-1}+f\left(u_{\varepsilon}\right)=0 & \text { in } \Omega, \\ \frac{\partial v_{\varepsilon}}{\partial n}=0 & \text { on } \partial \Omega\end{cases}
$$

Note that 


$$
\begin{aligned}
u_{\varepsilon}^{m-1}-f\left(u_{\varepsilon}\right)-u_{\varepsilon}^{-m-1}+f\left(\bar{u}_{\varepsilon}\right) & =\left(\bar{u}_{\varepsilon}+v_{\varepsilon}\right)^{m-1}-u_{\varepsilon}^{-m-1}-f\left(\bar{u}_{\varepsilon}+v_{\varepsilon}\right)+f\left(\bar{u}_{\varepsilon}\right) \\
& =\left[(m-1) \int_{0}^{1}\left(\bar{u}_{\varepsilon}+t v_{\varepsilon}\right)^{m-2} d t-\int_{0}^{1} f^{\prime}\left(\bar{u}_{\varepsilon}+t v_{\varepsilon}\right) d t\right] v_{\varepsilon}
\end{aligned}
$$

Then the first equation in (10) can be expressed as

$\varepsilon^{m} \Delta_{m} v_{\varepsilon}-\left[(m-1) \int_{0}^{1}\left(\bar{u}_{\varepsilon}+t v_{\varepsilon}\right)^{m-2} d t-\int_{0}^{1} f^{\prime}\left(\bar{u}_{\varepsilon}+t v_{\varepsilon}\right) d t\right] v_{\varepsilon}=\bar{u}_{\varepsilon}^{m-1}-f\left(\bar{u}_{\varepsilon}\right)$.

Multiplying both sides of (11) by $v_{\varepsilon}$, integrating over $\Omega$ and using integration by parts we have

$$
\varepsilon^{m} \int_{\Omega}\left|\nabla v_{\varepsilon}\right|^{m} d x+\int_{\Omega}\left[(m-1) \int_{0}^{1}\left(\bar{u}_{\varepsilon}+t v_{\varepsilon}\right)^{m-2} d t-\int_{0}^{1} f^{\prime}\left(\bar{u}_{\varepsilon}+t v_{\varepsilon}\right) d t\right] v_{\varepsilon}^{2} d x=0 .
$$

Since $0<\bar{u}_{\varepsilon}+t v_{\varepsilon} \leq 2 \max _{\Omega} u_{\varepsilon}(x) \leq C$ and $\lim _{t \rightarrow 0^{+}} \frac{f^{\prime}(t)}{t^{m-2}}=0$ for $m>2$ it follows that

$$
\left|\left((m-1)\left(\bar{u}_{\varepsilon}+t v_{\varepsilon}\right)^{m-2}-f^{\prime}\left(\bar{u}_{\varepsilon}+t v_{\varepsilon}\right)\right)_{-}\right| \leq C \quad \text { for }(x, t) \in \bar{\Omega} \times[0,1],
$$

where $(v)_{-}=\min \{v, 0\}$. Thus we get

$$
\varepsilon^{m} \int_{\Omega}\left|\nabla v_{\varepsilon}\right|^{m} d x \leq C \int_{\Omega} v_{\varepsilon}^{2} d x .
$$

On the other hand, (9) implies that the following Poincaré inequality holds for $v_{\varepsilon}$ with some positive constant $\gamma$ :

$$
\int_{\Omega}\left|\nabla v_{\varepsilon}\right|^{m} d x \geq \gamma^{m} \int_{\Omega}\left|v_{\varepsilon}\right|^{m} d x
$$

. Thus by Hölder inequality we have

$$
(\varepsilon \gamma)^{m} \int_{\Omega}\left|v_{\varepsilon}\right|^{m} d x \leq C\left(\int_{\Omega}\left|v_{\varepsilon}\right|^{m} d x\right)^{\frac{2}{m}},
$$

which yields

$$
\left\|v_{\varepsilon}\right\|_{L^{m}(\Omega)} \leq C \varepsilon^{-\frac{m}{m-2}}
$$

Therefore by interpolation and the fact that $\left\|v_{\varepsilon}\right\|_{L^{\infty}}(\Omega) \leq C$ it follows that for all $q \geq m$

$$
\left\|v_{\varepsilon}\right\|_{L^{q}(\Omega)} \leq C \varepsilon^{-\frac{m^{2}}{q(m-2)}} .
$$


Especially we have

$$
\left\|\left|v_{\varepsilon}\right|^{m-2} v_{\varepsilon}\right\|_{L^{\frac{N}{m-1}(\Omega)}} \leq C \varepsilon^{-\frac{m^{2}(m-1)}{N(m-2)}} .
$$

\section{Lemma 1}

The following estimate holds for $\mathrm{v}_{\varepsilon}$ :

$$
\left\|v_{\varepsilon}\right\|_{L^{\infty}(\Omega)} \leq C \varepsilon^{-\beta}
$$

where $\beta=\min \left\{\frac{m^{2}}{N(m-2)}, \frac{m}{m-1}\right\}$ and $\mathrm{C}$ is a positive constant depending only on $\mathrm{N}, \mathrm{m}, \Omega$ and the uniform upper bound as stated in Theorem A.

Proof We can rewrite (10) as follows.

$$
\begin{cases}\Delta_{m} v_{\varepsilon}-\left|v_{\varepsilon}\right|^{m-2} v_{\varepsilon}=-\left|v_{\varepsilon}\right|^{m-2} v_{\varepsilon}+\frac{1}{\varepsilon^{m}}\left(u_{\varepsilon}^{m-1}-f\left(u_{\varepsilon}\right)\right) & \text { in } \Omega, \\ \frac{\partial v_{\varepsilon}}{\partial n}=0 & \text { on } \partial \Omega .\end{cases}
$$

For convenience, let $g=-\left|v_{\varepsilon}\right|^{m-2} v_{\varepsilon}+\frac{1}{\varepsilon^{m}}\left(u_{\varepsilon}^{m-1}-f\left(u_{\varepsilon}\right)\right)$. It is easy to check $g \in L^{\frac{N}{m-1}}(\Omega)$ due to (14) and uniform boundedness of $u_{\varepsilon}$ as stated in Theorem A. Without loss of generality we

assume $\|g\|_{L^{\frac{N}{m-1}(\Omega)}} \neq 0$, or else the lemma holds trivially. Let $\tilde{v}_{\varepsilon}=v_{\varepsilon} /\|g\|_{L^{\frac{N}{m-1}(\Omega)}}^{\frac{1}{m-1}}$ and $\tilde{g}=g /\|g\|_{L^{\frac{N}{m-1}(\Omega)}}$. We can find that $\tilde{v}_{\varepsilon}$ satisfies the following equations

$$
\begin{cases}\Delta_{m} \tilde{v}_{\varepsilon}-\left|\tilde{v}_{\varepsilon}\right|^{m-2} \tilde{v}_{\varepsilon}=\tilde{g} & \text { in } \Omega, \\ \frac{\partial \tilde{v}_{\varepsilon}}{\partial n}=0 & \text { on } \partial \Omega .\end{cases}
$$

Next we use Moser iteration method to prove this lemma. For convenience, write $v$ instead of $\bar{v}_{\varepsilon}$.

If we multiply both sides of the first equation in (16) by $|v|^{m(s-1)} v(s \geq 1)$ and integrate over $\Omega$, then by virtue of the homogeneous Neumann boundary condition we have

$\left.\left.s^{-m}[m(s-1)+1] \int_{\Omega}|\nabla| v\right|^{s}\right|^{m} d x+\int_{\Omega}|v|^{m s} d x=-\int_{\Omega} \tilde{g}|v|^{m(s-1)} v d x$.

Since $s^{-m}[m(s-1)+1] \leq m+1$ for $s \geq 1$ and $\|\tilde{g}\|_{L^{\frac{N}{m-1}(\Omega)}}=1$ and we obtain from (17) that $s^{-m}[m(s-1)+1] \int_{\Omega}\left(\left.\left.|\nabla| v\right|^{s}\right|^{m}+|v|^{m s}\right) d x \leq(m+1)\left(\int_{\Omega}|v|^{\frac{[m(s-1)+1] N}{N-m+1}} d x\right)^{\frac{N-m+1}{N}}$.

Recall the Sobolev embedding theorem 


$$
\left(\int_{\Omega}|w|^{v} d x\right)^{\frac{m}{v}} \leq \Gamma^{m} \int_{\Omega}\left(|\nabla w|^{m}+|w|^{m}\right) d x
$$

for $w \in W^{1, m}(\Omega)$, where $\Gamma>0$ is a constant depending on $\Omega$.

It follows from (18) that

$$
s^{-m}[m(s-1)+1] \Gamma^{-m}\left(\int_{\Omega}|v|^{\nu s} d x\right)^{\frac{m}{v}} \leq(m+1)\left(\int_{\Omega}|\nu|^{\frac{[m(s-1)+1] N}{N-m+1}} d x\right)^{\frac{N-m+1}{N}} .
$$

Noting that $s^{-m}[m(s-1)+1] \geq s^{-(m-1)}$ for $s \geq 1$ thus we have

$$
\left.\left(\int_{\Omega}|v|^{\nu s} d x\right)^{\frac{m N}{v(N-m+1)}} \leq((m+1)) \Gamma^{m} s^{m-1}\right)^{\frac{N}{N-m+1}} \int_{\Omega}|v|^{\frac{[m(s-1)+1] N}{N-m+1}} d x
$$

for $s \geq 1$. Here we remark that if we take $s=1$ in (17) and take care of $\|\tilde{g}\|_{L^{\frac{N}{m-1}(\Omega)}}=1$ we have

$$
\int_{\Omega}\left(|\nabla v|^{m}+|v|^{m}\right) d x \leq|\Omega|^{\frac{(m-1)(N-m)+m}{N m}}\|v\|_{L^{v}(\Omega)},
$$

which yields from the Sobolev embedding theorem that

$$
\int_{\Omega}|v|^{v} d x \leq C_{0}
$$

$$
\text { with } C_{0}=\left(\Gamma^{m}|\Omega| \frac{(m-1)(N-m)+m}{N m}\right)^{\frac{v}{m-1}} \text {. }
$$

Next we define two sequences $\left\{s_{j}\right\}$ and $\left\{M_{j}\right\}$ by

$$
\begin{aligned}
{\left[m\left(s_{0}-1\right)+1\right] N /(N-m+1) } & =v, \\
{\left[m\left(s_{j+1}-1\right)+1\right] N /(N-m+1) } & =v s_{j} \text { for } j=0,1,2, \ldots, \\
M_{0} & =C_{0}, \\
M_{j+1} & =\left(\left[(m+1) \Gamma^{m} s_{j}^{m-1}\right]^{\frac{N}{N-m+1}} M_{j}\right)^{\frac{v(N-m+1)}{m N}} \text { for } j=0,1,2, \ldots,
\end{aligned}
$$

We note that $s_{j}$ can be explicitly given by

$$
\begin{aligned}
s_{j}= & \left(\frac{1}{N-m}+\frac{(m-1)(N-m)+m}{m}\right)\left[\left(\frac{N-m+1}{N-m}\right)^{j}-1\right] \\
& +\frac{N-m+1}{N-m}
\end{aligned}
$$

for $j=0,1,2, \ldots$, and it follows that $s_{j}>1$ for all $j \geq 0$ and $s_{j} \rightarrow \infty$ as $j \rightarrow \infty$. 
We shall show that

$$
\int_{\Omega}|v|^{\frac{\left[m\left(s_{j}-1\right)+1\right] N}{N-m+1}} d x \leq M_{j} \quad \text { for } \quad j \geq 0
$$

and

$$
M_{j} \leq e^{K s_{j-1}}
$$

for some constant $K>0$.

Clearly (25) holds for $j=0$ due to (21). Suppose that we have proved (25) for $j \geq 0$. Then by (20) we have

$$
\begin{aligned}
\int_{\Omega}|v|^{\frac{\left[m\left(s_{j+1}-1\right)+1\right] N}{N-m+1}} d x & \leq\left(\left[(m+1) \Gamma^{m} s_{j}^{m-1}\right]^{\frac{N}{N-m+1}} \int_{\Omega}|v|^{\frac{\left[m\left(s_{j}-1\right)+1\right] N}{N-m+1}} d x\right)^{\frac{v(N-m+1)}{m N}} \\
& \leq\left(\left[(m+1) \Gamma^{m} s_{j}^{m-1}\right]^{\frac{N}{N-m+1}} M_{j}\right)^{\frac{v(N-m+1)}{m N}} \\
& =M_{j+1},
\end{aligned}
$$

so that $(25)$ is true also for $j+1$. Therefore it remains to show (26).

Put $\sigma_{j}=\frac{v}{m} \log \left((m+1) \Gamma^{m} s_{j}^{m-1}\right)$ and $\mu_{j}=\log M_{j}$. Hence

$$
\mu_{j+1}=\frac{N-m+1}{N-m} \mu_{j}+\sigma_{j}
$$

Then from (24) it follows that

$$
\begin{aligned}
\sigma_{j}= & \frac{v}{m} \log \left((m+1) \Gamma^{m}\right) \\
+ & \frac{v(m-1)}{m} \log \left(\left(\frac{1}{N-m}+\frac{(m-1)(N-m)+m}{m}\right)\right. \\
& \left.\times\left[\left(\frac{N-m+1}{N-m}\right)^{j}-1\right]+\frac{N-m+1}{N-m}\right)
\end{aligned}
$$

and therefore we can find a constant $C^{*}$ such that

$$
\sigma_{j} \leq C^{*}(j+1)
$$

for all $j \geq 0$. We now define $\left\{\tau_{j}\right\}$ by $\tau_{0}=\mu_{0}$ and

$$
\tau_{j+1}=\frac{N-m+1}{N-m} \tau_{j}+C^{*}(j+1) \quad \text { for } \quad j \geq 0 .
$$

Clearly $\mu_{j} \leq \tau_{j}$ for all $j \geq 0$. Moreover 


$$
\tau_{j}=\left(\frac{N-m+1}{N-m}\right)^{j}\left[\mu_{0}+C^{*}(N-m)(N-m+1)\right]-C^{*}(j+N-m+1)(N-m),
$$

we know there is a constant $K>0$ such that

$$
\tau_{j} \leq K s_{j-1}
$$

and then we obtain (26). Here we note that $K$ depends only on $\mu_{0}, m, N$ and $C^{*}$, whereas $\mu_{0}$ depends only on, $\Gamma, m, N$ and $|\Omega|$, and $C^{*}$ depends only on $m, N$ and $\Gamma$. Thus (25) and (26) are true. So (22), (25) and (26) tell us that

$$
\|v\|_{L^{v s_{j-1}(\Omega)}}^{v s_{j-1}} \leq e^{K s_{j-1}}
$$

and hence letting $j \rightarrow \infty$ we obtain

$$
\|v\|_{L^{\infty}(\Omega)} \leq e^{K / v}
$$

Note that $v$ stands for $\bar{v}_{\varepsilon}$. Therefore till now we have shown that there is a constant $C$ depends only on $m, N$, and $\Omega$ such that

$$
\left\|\tilde{v}_{\varepsilon}\right\|_{L^{\infty}(\Omega)} \leq C .
$$

From the relation $\tilde{v}_{\varepsilon}=v_{\varepsilon} /\|g\|_{L^{\frac{N}{m-1}(\Omega)}}^{\frac{1}{m-1}}$ it follows that

$$
\left\|v_{\varepsilon}\right\|_{L^{\infty}(\Omega)} \leq C\|g\|_{L^{\frac{N}{m-1}(\Omega)}}^{\frac{1}{m-1}}
$$

Note that (14) and Theorem A tell us that

$$
\begin{aligned}
\|g\|_{L^{\frac{N}{m-1}(\Omega)}} & \leq\left\|v v^{m-2}\right\|_{L^{\frac{N}{m-1}(\Omega)}}+\left\|\frac{1}{\varepsilon^{m}}\left(u_{\varepsilon}^{m-1}-f\left(u_{\varepsilon}\right)\right)\right\|_{L^{\frac{N}{m-1}(\Omega)}} \\
& \leq C \varepsilon^{-\frac{m^{2}(m-1)}{N(m-2)}}+C \varepsilon^{-m}|\Omega|^{\frac{m-1}{N}} \\
& \leq C \varepsilon^{-\tilde{\beta}}
\end{aligned}
$$

with $\tilde{\beta}=\min \left\{\frac{m^{2}}{N(m-2)}, m\right\}$. Thus it follows from (27) that

$$
\left\|v_{\varepsilon}\right\|_{L^{\infty}(\Omega)} \leq C \varepsilon^{-\beta}
$$

with $\beta=\frac{\tilde{\beta}}{m-1}=\min \left\{\frac{m^{2}}{N(m-2)}, \frac{m}{m-1}\right\}$. Proof of Lemma 1 is complete. 
Next is a lemma related to $C^{1, \alpha}$-estimate of $v_{\varepsilon}$.

\section{Lemma 2}

Let $\mathrm{w} \in \mathrm{W}^{1, \mathrm{~m}}(\Omega) \cap \mathrm{L}^{\infty}(\Omega)(1<\mathrm{m}<\infty)$ be a weak solution to the following problem:

$$
\begin{cases}\Delta_{m} w=h & \text { in } \Omega \\ \frac{\partial w}{\partial n}=0 & \text { on } \partial \Omega\end{cases}
$$

Then there exist two constants $\mathrm{C}>0$ and $\alpha \in(0,1)$ depending only on $\mathrm{m}, \mathrm{N}$ and $\Omega$ such that

$$
\|w\|_{C^{1, \alpha}(\bar{\Omega})} \leq C\left(\|w\|_{L^{\infty}(\Omega)}+\|h\|_{L^{\infty}(\Omega)}^{\frac{1}{m-1}}\right) .
$$

Proof In [16] the author proved the follows: Suppose $w \in W^{1, m}\left(B_{1}(0)\right) \cap L^{\infty}\left(B_{1}(0)\right)$ (hereafter $B_{R}(x)$ denotes the ball centered at $x$ with radius $R$ ) is a solution of the differential equation

$$
\int_{B_{1}(0)} \sum_{j=1}^{N}\left\{a_{j}(x, w, \nabla w) \cdot \psi_{x_{j}}\right\}-h \psi d x=0, \quad \forall \psi \in C_{c}^{\infty}\left(B_{1}(0)\right)
$$

with $\|w\|_{L^{\infty}}{ }_{\left(B_{1}(0)\right)},\|h\|_{L^{\infty}}{ }_{\left(B_{1}(0)\right)} \leq 1$ and for $j=1, \ldots, N$,

$$
a_{j} \in C^{0}\left(B_{1}(0) \times \mathbb{R} \times \mathbb{R}^{N}\right) \cap C^{1}\left(B_{1}(0) \times \mathbb{R} \times\left(\mathbb{R}^{N}-\{0\}\right)\right)
$$

and satisfies the following ellipticity and growth conditions

$$
\begin{aligned}
a_{j}(x, \mu, 0) & =0, \\
\sum_{i, j=1}^{N} \frac{\partial a_{j}}{\partial \eta_{i}}(x, \mu, \eta) \cdot \xi_{i} \xi_{j} & \geq \gamma_{1}|\eta|^{m-2} \cdot|\xi|^{2}, \\
\sum_{i, j=1}^{N}\left|\frac{\partial a_{j}}{\partial \eta_{i}}(x, \mu, \eta)\right| & \leq \Gamma_{1}|\eta|^{m-2}, \\
\sum_{i, j=1}^{N}\left|\frac{\partial a_{j}}{\partial x_{i}}(x, \mu, \eta)\right|+\left|\frac{\partial a_{j}}{\partial \mu}(x, \mu, \eta)\right| & \leq \Gamma_{1}|\eta|^{m-1},
\end{aligned}
$$

for some positive constants $\gamma_{1}, \Gamma_{1}$ and $x \in B_{1}(0), \mu \in \mathbb{R}, \eta \in \mathbb{R}^{N}-\{0\}$ and $\xi \in \mathbb{R}^{N}$. Then there exist constants $\alpha \in(0,1), C$ depending only on $m, N, \gamma_{1}$ and $\Gamma_{1}$ such that

$$
\|w\|_{C^{1, \alpha}\left(B_{\frac{1}{2}}(0)\right)} \leq C .
$$

Here we remark that the proof also works well if $C^{1} C^{1}\left(B_{1}(0) \times \mathbb{R} \times\left(\mathbb{R}^{N}-\{0\}\right)\right)$ is replaced by locally Lipschitz for $(x, \mu, \eta) \in B_{1}(0) \times \mathbb{R} \times\left(\mathbb{R}^{N}-\{0\}\right)$.

For any compact set $K_{\Omega} \subset \subset \Omega$ and $x_{0} \in K_{\Omega}$ let $\rho=\min \left\{\right.$ dist $\left.\left(K_{\Omega}, \partial \Omega\right), 1\right\}$. Applying above interior estimate to 


$$
\tilde{w}(x)=\frac{w\left(x_{0}+\rho x\right)}{\left(\|w\|_{L^{\infty}(\Omega)}+\left\|\rho^{m} h\right\|_{L^{\infty}(\Omega)}^{\frac{1}{(m-1)}}\right)}
$$

and using standard covering argument we have a constant $\alpha * \in(0,1)$ depending only on $m$ and $N$ such that

$$
\|\tilde{w}\|_{C^{1, \alpha_{*}\left(K_{\Omega}\right)}} \leq C
$$

with $C$ depending only on $m, N, K_{\Omega}$ and $\rho$, which tells us that

$$
\|\tilde{w}\|_{C^{1, \alpha_{*}\left(K_{\Omega}\right)}} \leq C\left(m, N, K_{\Omega}, \rho\right)\left(\|w\|_{L^{\infty}(\Omega)}+\|h\|_{L^{\infty}(\Omega)}^{\frac{1}{(m-1)}}\right) .
$$

For the boundary regularity we can use the local reflection method. For $P \in \partial \Omega$, without loss of generality, we may assume that $P$ is the coordinate origin and the $x_{N}$-axis is normal to $\partial \Omega$ at $P$, hence there exists a smooth function $h_{*}\left(x^{\prime}\right), x^{\prime}=\left(x_{1}, \ldots, x_{N-1}\right)$ defined for $\left|x^{\prime}\right|<\delta$ satisfying

$$
\begin{aligned}
& \left(\mathrm{f}_{1}\right): h *(0)=0 \text { and } \frac{\partial h_{*}}{\partial x_{j}}(0)=0 \text { for } j=1, \ldots, N-1 \text { and } \\
& \left(\mathrm{f}_{2}\right): \Omega \cap \mathcal{B}=\left\{\left(x^{\prime}, x_{N}\right) \mid x_{N}>h_{*}\left(x^{\prime}\right)\right\} \text { and } \partial \Omega \cap \mathcal{B}=\left\{\left(x^{\prime}, x_{N}\right) \mid x_{N}=h_{*}\left(x^{\prime}\right)\right\} \text { in a neighborhood } \\
& \mathcal{B} \text { of } P .
\end{aligned}
$$

For $y \in \mathbb{R}^{N}$ and $|y|$ sufficiently small, we define a mapping straightening the boundary portion around $P$ as in [11]: $\left.x=\Phi(y)=\left(\phi_{1} y\right), \ldots, \phi(y)\right)$ by

$$
\begin{aligned}
& \phi_{j}(y)=y_{j}-y_{N}\left(\frac{\partial h_{*}}{\partial y_{j}}\left(y^{\prime}\right)\right) \text { for }=1, \ldots, N-1, \\
& \phi_{N}(y)=y_{N}+h_{*}\left(y^{\prime}\right) .
\end{aligned}
$$

Since in virtue of property $\mathrm{f}_{1}$ the differential map $D \Phi$ of $\Phi$ satisfies $D \Phi(0)=I_{N \times N}$, the identity matrix, we can assume $\Phi$ is defined on $B_{\kappa}(0)$ for some sufficiently small constant $\kappa$ $>0$ such that

$$
\left\|D \Phi(y)-I_{N \times N}\right\| \leq \frac{1}{2}
$$

for all $y \in B_{\kappa}(0)$ where $\|A\|=\max _{\|x\|=1}(A x, x)$ for a $N \times N$ matrix $A$. Then $\Phi$ has an inverse mapping $y=\Psi(x)=\Phi^{-1}(x)$ in the neighborhood $\left(B_{\kappa}(0)\right)$ of $x=0$. We write $\Psi(x)=(\psi(x)$, $\ldots, \psi_{N}(x)$. Set $\widehat{w}(y)=w(\Phi(y))$ and 


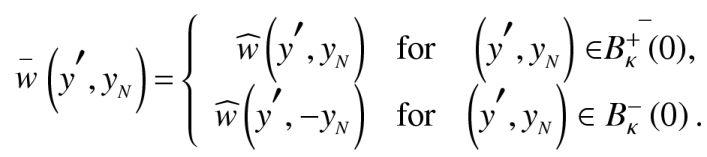

Let

$$
g i j=\sum_{k=1}^{N} \frac{\partial \phi_{k}}{\partial y_{i}} \frac{\partial \phi_{k}}{\partial y_{i}}(y), \quad g^{i j}=\sum_{k=1}^{N} \frac{\partial \psi_{i}}{\partial x_{k}} \frac{\partial \psi_{j}}{\partial x_{k}}(\Phi(y))
$$

Here we note that $g_{i N \mid B_{K}(0) \cap\left\{y_{N}=0\right\}}=0$ for $i=1, \ldots, N-1$. Next denote

$$
\begin{aligned}
& \bar{g} i j\left(y^{\prime}, y_{N}\right)=\left\{\begin{aligned}
g i j\left(y^{\prime}, y_{N}\right) & \text { for }\left(y^{\prime}, y_{N}\right) \in B_{\kappa}^{+}(0), \\
(-1)^{\delta_{i N}+\delta_{j N}} g i j\left(y^{\prime},-y_{N}\right) & \text { for }\left(y^{\prime}, y_{N}\right) \in B_{\kappa}^{-}(0),
\end{aligned}\right. \\
& g^{i j}\left(y^{\prime}, y_{N}\right)=\left\{\begin{aligned}
g^{i j}\left(y^{\prime}, y_{N}\right) & \text { for }\left(y^{\prime}, y_{N}\right) \in B_{K}^{+}(0), \\
(-1)^{\delta_{i N}+\delta_{j N}} g^{i j}\left(y^{\prime},-y_{N}\right) & \text { for }\left(y^{\prime}, y_{N}\right) \in B_{K}^{-}(0),
\end{aligned}\right.
\end{aligned}
$$

where $\delta_{i j}$ is the Kronecker symbol. Denote $g_{*}(y)=\operatorname{det}\left(\bar{g}_{i j}(y)\right)$. Then from (31) we know

$$
\sqrt{g_{*}(y)} \leq C(N), \quad \forall y \in B_{\kappa}(0)
$$

for some absolute constant $C(N)>0$. Then $\tilde{w} \in W^{1, m}\left(B_{\kappa}(0)\right) \cap L^{\infty}\left(B_{\kappa}(0)\right)$ satisfies

$$
\left\{\begin{array}{l}
\sum_{j=1}^{N} \frac{\partial}{\partial y_{j}} a_{j}(y, \nabla \bar{w})=\sqrt{g_{*}}(h \circ \Phi) \quad \text { in } B_{K}(0) \\
\frac{\partial \bar{w}}{\partial y_{N}}=0 \quad \text { on } \quad B_{K}(0) \cap\left\{y_{N}=0\right\}
\end{array}\right.
$$

with

$a_{j}(y, \nabla \bar{w})=\sum_{i=1}^{N}\left[\sum_{s, l}^{N} g^{-s l} \frac{\partial \bar{w}}{\partial y_{s}} \frac{\partial \bar{w}}{\partial y_{l}}\right]^{\frac{m-2}{2}} \quad{\sqrt{g_{*}}}^{-j} \frac{\partial \bar{w}}{\partial y_{i}}, \quad$ for $\quad j=1, \ldots, N$

It is not hard to check that $a_{j}(y, \eta)(j=1, \ldots, N)$ satisfies all inequalities in (29) with some positive constants $\gamma_{1}$ depending only on $m$ and $N$ due to (31) and (33) and $\Gamma_{1}$ depending only on $m, N, D^{2} \Phi_{i}$ and $D^{2} \psi_{i}(i=1, \ldots, N)$ via $\frac{\partial \bar{s}_{s l}}{\partial y_{j}}$ and $\frac{\partial g^{-s l}}{\partial y_{j}}(s, l, j=1, \ldots, N)$ (hence $\Gamma_{1}$ depends only on $m, N$ and $\Omega$ ).

Applying the interior estimate to 


$$
\tilde{\bar{w}}(y)=\frac{\bar{w}(\kappa y)}{\|\bar{w}\|_{L^{\infty}\left(B_{K}(0)\right)}+\left\|\kappa^{m} \sqrt{g_{*}}(h \circ \Phi)\right\|_{L^{\infty}\left(B_{K}(0)\right)}^{\frac{1}{m-1}}}
$$

we know there exist two constants $\alpha_{0} \in(0,1)$ and $C$ depending only on $m, N$ and $\Omega$ such that

$$
\|\tilde{\bar{w}}\|_{C^{1, \alpha_{0}}\left(B_{\frac{1}{2}}(0)\right)} \leq C
$$

which tells us that

$$
\|\bar{w}\|_{C^{1, \alpha_{0}}\left(\Phi\left(B_{\frac{K}{2}}^{-}(0)\right)\right)} \leq C(m, N, \kappa, \Omega)\left(\|w\|_{L^{\infty}(\Omega)}+\|f\|_{L^{\infty}(\Omega)}^{\frac{1}{m-1}}\right) .
$$

Especially, we have

$$
\|w\|_{C^{1, \alpha_{0}}\left(\Phi\left(B_{\frac{K^{-}}{2}}^{-}(0)\right)\right)} \leq C(m, N, \kappa, \Omega)\left(\|w\|_{L^{\infty}(\Omega)}+\|f\|_{L^{\infty}(\Omega)}^{\frac{1}{m-1}}\right) .
$$

Since $\partial \Omega$ is compact Lemma 2 follows from standard covering argument to patch the interior estimate (30) and the boundary estimate (37) together. Proof of Lemma 2 is complete.

With helps of Lemma 1 and Lemma 2 we can give proof of Theorem 1 as follows.

Proof of Theorem 1 It follows from (10), Lemma 1 and Lemma 2 that there exist a positive constant $\alpha \in(0,1)$ depending only on $m, N, \Omega$ such that

$$
\begin{aligned}
\left\|v_{\varepsilon}\right\|_{C^{1, \alpha}(\bar{\Omega})} & \leq C\left(\left\|v_{\varepsilon}\right\|_{L^{\infty}(\Omega)}+\left\|\frac{1}{\varepsilon^{m}}\left(u_{\varepsilon}^{m-1}-f\left(u_{\varepsilon}\right)\right)\right\|_{L^{\infty}(\Omega)}^{\frac{1}{m-1}}\right) \\
& \leq C\left(\varepsilon^{-\beta}+\varepsilon^{-\frac{m}{m-1}}\right) \leq C_{\varepsilon}^{-\beta},
\end{aligned}
$$

with $\beta=\min \left\{\frac{m^{2}}{N(m-2)}, \frac{m}{m-1}\right\}$ as defined in Lemma 1. that we have used uniform boundedness of $u_{\varepsilon}$ as stated in Theorem A. Therefore the constant $C$ depends only on $m, N$, $\Omega, f$ and the uniform bound of $u_{\varepsilon}$ as stated in Theorem A.

Let $Z_{f}=\min \left\{t>0 \mid t^{m-1}=\mathrm{f}(\mathrm{t})\right\}$. It follows from assumption $\left(\mathrm{f}_{2}\right)$ that $Z_{f}>0$. The maximum principle tells us that $\sup _{\Omega} u_{\varepsilon} \geq Z_{f}$ ([8]). Thus it follows from (38) and Theorem $\mathrm{A}$ that for sufficiently large $\varepsilon$,

$$
\frac{1}{2} Z_{f} \leq \bar{u}_{\varepsilon} \leq C
$$


which implies there exist a sequence $\left\{\varepsilon_{j}\right\}$ with $\lim _{j \rightarrow \infty} \varepsilon_{j}=\infty$ and a constant $u_{c}>0$ such that $\lim _{j \rightarrow \infty} \bar{u}_{\varepsilon_{j}}=u_{c}$. Note that from (1)-(3) it follows that

$$
\varepsilon_{j}^{m} \int_{\Omega}\left|\nabla u_{\varepsilon_{j}}\right|^{m} d x=\int_{\Omega} u_{\varepsilon_{j}}\left(f\left(u_{\varepsilon_{j}}\right)-u_{\varepsilon_{j}}^{m-1}\right) d x
$$

Note that (12) and (38) imply

$$
\varepsilon_{j}^{m} \int_{\Omega}\left|\nabla u_{\varepsilon_{j}}\right|^{m} d x=\varepsilon_{j}^{m} \int_{\Omega}\left|\nabla v_{\varepsilon_{j}}\right|^{m} d x \leq C \int_{\Omega} v_{\varepsilon_{j}}^{2} d x \leq C \varepsilon_{j}^{-2 \beta}|\Omega| .
$$

Taking limit at both sides of (40) as $j \rightarrow \infty$ and using (38) we obtain

$$
\int_{\Omega} u_{c}\left(f\left(u_{c}\right)-u_{c}^{m-1}\right) d x=0
$$

which implies $f\left(u_{c}\right)-u_{c}^{m-1}=0$, i.e., the constant $u_{c}$ is a positive zero of $t^{m-1}-f(t)$. Proof of Theorem 1 is complete.

\section{Uniform upper bound for least energy solutions of the critical case}

In this section we first discuss the existence of least energy solutions of the critical case

$f(u)=u_{+}^{\frac{N(m-1)+m}{N-m}}(1<m<N)$ and then show that for $\varepsilon \in[1, \infty)$ there is an upper bound independent of $\varepsilon$ (hence uniform) for the least energy solutions.

Note that for this critical case the problem (1)-(3) is as follows.

$$
\begin{cases}\varepsilon^{m} \lambda_{m} u-u^{m-1}+u^{\frac{N(m-1)+m}{N-m}}=0 & \text { in } \Omega \\ u>0 & \text { in } \Omega, \\ \frac{\partial u}{\partial n}=0 & \text { on } \partial \Omega,\end{cases}
$$

Let $u=\varepsilon^{\frac{N-m}{m}} v$. Then $v$ solves the following problem:

$$
\begin{cases}\Delta_{m} v-\frac{1}{\varepsilon^{m}} v^{m-1}+v^{\frac{N(m-1)+m}{N-m}}=0 & \text { in } \Omega \\ u>0 & \text { in } \Omega, \\ \frac{\partial u}{\partial n}=0 & \text { on } \partial \Omega,\end{cases}
$$

Associated with (45)-(47) is functional $J^{\varepsilon}: W^{1, m}(\Omega) \rightarrow \mathbb{R}$ defined by

$$
J^{\varepsilon}(v)=\frac{1}{m} \int_{\Omega}\left(|\nabla v|^{m}+\frac{1}{\varepsilon^{m}}|v|^{m}\right) d x-\frac{N-m}{N m} \int_{\Omega} v_{+}^{v} d x .
$$

Recall that $J_{\varepsilon}: W^{1, m}(\Omega) \rightarrow \mathbb{R}$ was defined by (4) and also note that for $u=\varepsilon^{\frac{N-m}{m}} v \in W^{1, m}(\Omega)$ we have

$$
J^{\varepsilon}(u)=\varepsilon^{N} J^{\varepsilon}(v)
$$


Thus existence of least energy solutions of (45)-(47) implies the existence of least energy solution of (42)-(44).

Recall $\Gamma_{\text {set }}$ defined by (5) and let

$$
\tilde{c}_{\varepsilon}=\inf _{h \in \Gamma_{\mathrm{set}} 0 \leq t \leq 1} \max ^{\varepsilon}(h(t)) .
$$

The following result was proved in [18] (see also [15]) for $1<m<N$ and also see [17] for $m$ $=2$.

Lemma $30<\tilde{c}_{\varepsilon} \leq \frac{1}{2 N} S_{m}^{\frac{N}{m}}$ is a critical value of the functional $J^{\varepsilon}$ defined by (48), where

$$
S_{m}=\inf _{w \in C_{c}^{\infty}\left(\mathbb{R}^{N}\right)}\left\{\int_{\mathbb{R}^{N}}|\nabla w|^{m} d x: \int_{\mathbb{R}^{N}}|w|^{\nu}=1\right\}
$$

is the best Sobolev constant for the embedding $\mathbb{D}^{1, m}\left(\mathbb{R}^{N}\right) \hookrightarrow L^{v}\left(\mathbb{R}^{N}\right)$, and

$\mathbb{D}^{1, m}\left(\mathbb{R}^{N}\right)=\left\{\nabla w \in L^{m}\left(\mathbb{R}^{N}\right): w \in L^{v}\left(\mathbb{R}^{N}\right)\right\}_{\text {with norm }}\left(\int_{\mathbb{R}^{N}}|\nabla w|^{m} d x\right)^{\frac{1}{m}}$.

From above discussions we know there is a least energy solution $u_{\varepsilon}$ to the problem (42)-(44) with

$$
J_{\varepsilon}\left(u_{\varepsilon}\right) \leq \frac{1}{2 N} \varepsilon^{N} S_{m}^{\frac{N}{m}}
$$

Note that due to the characterization (7) we also know

$$
J_{\varepsilon}\left(u_{\varepsilon}\right) \leq M_{*}[1]=\frac{1}{N}|\Omega| .
$$

Therefore (51) and (52) together imply

$$
J_{\varepsilon}\left(u_{\varepsilon}\right)=\frac{1}{N} \int_{\Omega} u_{\varepsilon}^{v} d x \leq C_{1} .
$$

with $C_{1}=\min \left\{\frac{1}{2 N} \varepsilon^{N} S_{m}^{\frac{N}{m}}, \frac{1}{N}|\Omega|\right\}$. Also note that

$$
\int_{\Omega}\left(\varepsilon^{m}\left|\nabla u_{\varepsilon}\right|^{m}+u_{\varepsilon}^{m}\right) d x=\int_{\Omega} u_{\varepsilon}^{v} d x \leq C_{1} N
$$

Next we give proof of Theorem 2 .

Proof of Theorem 2 We need several steps to prove this theorem.

\section{Claim 1}

There exists a constant $\mathrm{C}_{*}>0$ such that 


$$
\sup _{\Omega} u_{\varepsilon} \leq C_{*} \varepsilon^{\frac{N-m}{m}}
$$

Suppose not. Then there exist a sequence $\left\{\varepsilon_{\mathrm{k}}\right\}(\mathrm{k} \geq 1)$, a sequence of constants $\left\{\mathrm{c}_{\mathrm{k}}\right\}$ and a sequence of points $\left\{P_{k}\right\} \subset \bar{\Omega}$ with $\varepsilon_{\mathrm{k}}, \mathrm{C}_{\mathrm{k}} \rightarrow \infty$ such that

$$
\tilde{M}_{k}=\sup _{\Omega} u_{\varepsilon_{k}}=u_{\varepsilon_{k}}\left(P_{k}\right) \leq c_{k} \varepsilon_{k}^{\frac{N-m}{m}} .
$$

Since $\bar{\Omega}$ is compact, passing by a subsequence if it is necessary we can assume $P_{k} \rightarrow P_{0} \in \bar{\Omega}$. For convenience, we write $\mathrm{u}_{\mathrm{k}}$ instead of $\mathrm{u}_{\varepsilon_{\mathrm{k}}}$.

\section{Case 1}

$P_{0} \in \partial \Omega$. Without loss of generality we may assume that $P_{0}$ is the coordinate origin and the $x_{\mathrm{N}}$-axis is normal to $\partial \Omega$ at $P_{0}$. As in the proof of Lemma 2 we introduce a mapping $\Phi$ straightening the boundary portion around $P_{0}$ which is defined on $B_{\kappa}(0)$ for some constant $\kappa$ $>0$. Let $\bar{u}_{k}$ be defined as in the proof of Lemma 2 with $w$ replaced by $u_{k}$. We know $\bar{u}_{k} \in W^{1, m}\left(B_{k}(0)\right)$ satisfies

$$
\begin{cases}\varepsilon^{m} \sum_{j=1}^{N} \frac{\partial}{\partial y_{j}} a_{j}\left(y, \nabla \bar{u}_{k}\right)=\sqrt{g_{*}}\left(\bar{u}_{k}^{m-1}-\bar{u}_{k}^{-\frac{N(m-1)+m}{N-m}}\right) & \text { in } B_{K}(0) \\ \frac{\partial \bar{u}_{k}}{\partial y_{N}}=0 & \text { on } B_{\kappa}(0) \cap\left\{y_{N}=0\right\}\end{cases}
$$

with

$$
a_{j}\left(y, \nabla \bar{u}_{k}\right)=\sum_{i=1}^{N}\left[\sum_{s, l}^{N} g^{-s l} \frac{\partial \bar{u}_{k}}{\partial y_{s}} \frac{\partial \bar{u}_{k}}{\partial y_{l}}\right]^{\frac{m-2}{2}}{\sqrt{g_{*}}}_{g}^{-j i} \frac{\partial \bar{u}_{k}}{\partial y_{i}},
$$

for $j=1, \ldots, N$, where $g^{-s l}$ is defined as in the proof of Lemma 2.

Put $Q_{k}=\Psi\left(P_{k}\right)$ with $\Psi$ being the inverse of $\Phi$ as defined in the proof of Lemma 2 and write $Q_{k}=\left(q_{k}^{\prime}, \alpha_{k}\right), \alpha_{k} \geq 0$. Since $Q_{k} \rightarrow 0$ as $k \rightarrow \infty$ we may assume that $\left|Q_{k}\right| \leq \frac{\kappa}{2}$ for all $k$ with $\kappa$ being chosen as in the proof of Lemma 2 .

Let $\lambda_{k}=\varepsilon_{k} / \tilde{M}_{k}^{\frac{m}{N-m}}$. From (56) it follows that $\lambda_{k} \leq 1 / c_{k}^{\frac{m}{N-m}} \rightarrow 0$ as $k \rightarrow \infty$. Then the proof can be divided to treat two cases according to the behavior of $\frac{\alpha_{k}}{\lambda_{k}}$ as $k \rightarrow \infty$.

Subcase (i). The sequence $\left\{\frac{\alpha_{k}}{\lambda_{k}}\right\}$ remain bounded. By passing to a subsequence if it is necessary we may assume 


$$
\frac{\alpha_{k}}{\lambda_{k}} \rightarrow \beta_{*} \geq 0 \quad \text { as } \quad k \rightarrow \infty
$$

Define a scaled function by

$$
v_{k}(z)=\tilde{M}_{k}^{-1} \bar{u}_{k}\left(\lambda_{k} z^{\prime}+q_{k}^{\prime}, \lambda_{k} z_{N}\right)
$$

Note that $v_{k}$ is well-defined in the ball $B_{\frac{k}{\lambda_{k}}}$ and that $0<v_{k}(z) \leq 1$ for all $k$. From (57) we see that $v_{k}$ satisfies

$$
\left\{\begin{array}{l}
\sum_{j=1}^{N} \frac{\partial}{\partial z_{j}} \tilde{a}_{j}\left(z, \nabla v_{k}\right)=\sqrt{\tilde{g}_{*}}\left(\tilde{M}_{k}^{m-v} v_{k}^{m-1}-v_{k}^{\frac{N(m-1)+m}{N-m}}\right) \text { in } B_{\frac{\kappa}{\lambda_{k}}}(0), \\
\frac{\partial v_{k}}{\partial z_{N}}=0 \quad \text { on } \quad B_{\frac{\kappa}{\lambda_{k}}}(0) \cap\left\{z_{n}=0\right\}
\end{array}\right.
$$

with

$$
\tilde{a}_{j}\left(z, \nabla v_{k}\right)=\sum_{i=1}^{N}\left[\sum_{s, l}^{N} \tilde{q}^{g}(z) \frac{\partial v_{k}}{\partial z_{s}} \frac{\partial v_{k}}{\partial z_{l}}\right]^{\frac{m-2}{2}} \sqrt{\tilde{g}_{*}(z) \check{g}^{j i}}(z) \frac{\partial v_{k}}{\partial z_{i}},
$$

for $j=1, \ldots, N$, where ${ }^{\frac{\tilde{g}^{s l}}{g}}(z)=\bar{g}^{-s l}\left(\lambda_{k} z^{\prime}+q_{k}{ }^{\prime}, \lambda_{k} z_{N}\right), \tilde{g}_{*}(z)=g_{*}\left(\lambda_{k} z^{\prime}+q_{k}^{\prime}, \lambda_{k} z_{N}\right)$ for $s, l=1, \ldots, N$. Note that $\tilde{a}_{j}\left(z, \nabla v_{k}\right)$ satisfies the structure conditions (29) with two positive constants $\gamma_{1}=\gamma_{1}$ $(m, N)$ and $\Gamma_{1}=\Gamma_{1}(m, N, \Omega)$ (see the proof of Lemma 2$)$. Next choose a sequence $\left\{R_{l}\right\}$ such that $R_{l} \rightarrow \infty$ as $l \rightarrow \infty$. For fixed $l, B_{4 R_{l}}(0) \subset B_{\frac{\kappa}{\lambda_{k}}}(0)$ provided $k$ is sufficiently large. Thus by the interior estimate we mentioned at the beginning of the proof of Lemma 2 it follows that $\left\{v_{k}\right\}$ is uniformly bounded in $C^{1, \alpha}\left(B_{2 R_{l}}(0)\right)$ for some $\alpha \in(0,1)$ which is independent of $k$. By standard arguments using a diagonal process, we can find a subsequence $\left\{v_{k_{j}}\right\}$ which converge uniformly to $\tilde{v} \in C^{1, \alpha^{\#}}\left(\mathbb{R}^{N}\right)\left(0<\alpha^{\#}<\alpha\right)$ on any compact subset of $\mathbb{R}^{N}$. Note that $\underline{\sim} \underline{\underline{g}}$ $\bar{g}(z) \rightarrow \delta_{i j}$ as $k \rightarrow \infty$ due to the fact that $\lambda_{k} \rightarrow 0$ and $D \Psi(x) \rightarrow D \Psi(0)=I_{\mathrm{N} \times \mathrm{N}}$ as $x \rightarrow 0$. Also note that $\tilde{M}_{k} \rightarrow \infty$ as $k \rightarrow \infty$. Thus we conclude that $\tilde{v}$ satisfies

$$
\left\{\begin{array}{l}
\Delta_{m} \tilde{v}+\tilde{v}^{\frac{N(m-1)+m}{N-m}}=0 \quad \text { in } \mathbb{R}^{N} \\
0 \leq \tilde{v} \leq 1, \quad \tilde{v}\left(0, \ldots, 0, \beta_{*}\right)=1
\end{array}\right.
$$

On the other hand, for any fixed $R>\beta *+1$ we have 


$$
\begin{aligned}
\int_{B_{R}(0)}|\nabla \bar{v}|^{m} d z & =\lim _{j \rightarrow \infty} \int_{B_{R}(\mathbf{0})}\left|\nabla v_{k_{j}}\right|^{m} d z \leq \lim _{j \rightarrow \infty} \int_{B \frac{k}{\lambda_{k_{j}}}(0)}\left|\nabla v_{k_{j}}\right|^{m} d z \\
& =\lim _{j \rightarrow \infty} \varepsilon_{k_{j}}^{m-N} \int_{B_{k}(\mathbf{0})}\left|\nabla \bar{u}_{k_{j}}\right|^{m} d y \\
& =\lim _{j \rightarrow \infty} 2 \varepsilon_{k_{j}}^{m-N} \int_{B_{k}^{+}(0)}\left|\nabla \bar{u}_{k_{j}}\right|^{m} d y \\
& =\lim _{j \rightarrow \infty} 2 \varepsilon_{k_{j}}^{m-N} \int_{\Phi\left(B_{k}^{+}(0)\right)}\left|\nabla u_{k_{j}}\right|^{m}|\operatorname{det}(D \Psi)| d x \\
& \leq \lim _{j \rightarrow \infty} \varepsilon_{k_{j}}^{-N}\left(C(N) \int_{\Omega} \varepsilon_{k_{j}}^{m}\left|\nabla u_{k_{j}}\right|^{m} d x\right)=0 \quad \text { (by }
\end{aligned}
$$

Hence $\nabla \tilde{v} \equiv 0$ in $B_{R}(0)$, which implies $\tilde{v} \not \equiv 1$ in $B_{R}(0)$ since $\tilde{v}\left(0, \ldots, 0, \beta_{*}\right)=1$, and this contradicts, (61).

Subcase (ii). The sequence $\left\{\frac{\alpha_{k}}{\lambda_{k}}\right\}$ is unbounded. We may assume that $\frac{\alpha_{k}}{\lambda_{k}} \rightarrow \infty$ as $k \rightarrow \infty$. In this case we define

$$
v_{k}(z)=\tilde{M}_{k}^{-1} \bar{u}_{k}\left(\lambda_{k} z+Q_{k}\right) .
$$

Then $v_{k}$ is well defined in the ball $B_{\frac{k}{\lambda_{k}}}(0)$ and $v_{k}(0)=1$ for all $k=1,2, \ldots$ From (58) we see that $v_{k}$ satisfies

$$
\begin{cases}\sum_{j=1}^{N} \frac{\partial}{\partial z_{j}} \tilde{a}_{j}\left(z, \nabla v_{k}\right)=\sqrt{\tilde{g}_{*}}\left(\tilde{M}_{k}^{m-v} v_{k}^{m-1}-v_{k}^{\frac{N(m-1)+m}{N-m}}\right) & \text { in } B_{\frac{\kappa}{\lambda_{k}}}(0), \\ \frac{\partial v_{k}}{\partial z_{N}}=0 & \text { on } B_{\frac{\kappa}{\lambda_{k}}}(0) \cap\left\{z_{n}=-\frac{\alpha_{k}}{\lambda_{k}}\right\}\end{cases}
$$

with

$$
\tilde{a}_{j}\left(z, \nabla v_{k}\right)=\sum_{i=1}^{N}\left[\sum_{s, l}^{N} \tilde{\bar{c}}^{s l} \text { (z) } \frac{\partial v_{k}}{\partial z_{s}} \frac{\partial v_{k}}{\partial z_{l}}\right]^{\frac{m-2}{2}} \sqrt{\tilde{g}^{\prime} *(z) \tilde{c}^{j i}} \quad(z) \frac{\partial v_{k}}{\partial z_{i}},
$$

for $j=1, \ldots, N$, where $\stackrel{\sim}{g}^{s l}(z)=\bar{g}^{s l}\left(\lambda_{k} z+Q_{k}\right), \tilde{g}_{*}(z)=g_{*}\left(\lambda_{k} z+Q_{k}\right)$ in $(63)$ for $s, l=1, \ldots, N$.

For any $R>0$ we have $\frac{\alpha_{k}}{\lambda_{k}}>2 R$ if $k$ is sufficiently large, so that the entire ball $B_{2 R}^{-}(0)$ is

contained in $B_{\frac{k}{\lambda_{k}}}(0) \cap\left\{z_{N}>-\frac{\alpha_{k}}{\lambda_{k}}\right\}$. Repeating the argument as in the subcase (i), we obtain a subsequence $v_{k}$ which converges uniformly to $C^{1, \alpha^{\#}}$ function $\bar{v}$ on any compact set of $\mathbb{R}^{N}$, and $\bar{v}$ satisfies

$$
\left\{\begin{array}{l}
\Delta_{m} \bar{v}+\bar{v}^{\frac{N(m-1)+m}{N-m}}=0 \quad \text { in } \mathbb{R}^{N} \\
0 \leq \bar{v} \leq 1, \quad \bar{v}(0)=1
\end{array}\right.
$$

Nevertheless, for any fixed $R>0$ we know 


$$
\begin{aligned}
\int_{B_{R}(0)}|\nabla \bar{v}|^{m} d z & =\lim _{j \rightarrow \infty} \int_{B_{R}(0)}\left|\nabla v_{k_{j}}\right|^{m} d z \leq \lim _{j \rightarrow \infty} \int_{B \frac{k}{\lambda_{k_{j}}}(0) n\left\{\left\{z_{N}>-\frac{\alpha_{k}}{\lambda_{k}}\right\}\right.}\left|\nabla v_{k_{j}}\right|^{m} d z \\
& =\lim _{j \rightarrow \infty} \varepsilon_{k_{j}}^{m-N} \int_{B_{k}^{+}(0)}\left|\nabla u_{k_{j}}\right|^{m} d y \\
& =\lim _{j \rightarrow \infty} \varepsilon_{k_{j}}^{m-N} \int_{\Phi\left(B_{k}^{+}(0)\right)}\left|\nabla u_{k_{j}}\right|^{m}|\operatorname{det}(D \Psi)| d x \\
& \leq \lim _{j \rightarrow \infty} \varepsilon_{k_{j}}^{-N}\left(C(N) \int_{\Omega} \varepsilon_{k_{j}}^{m}\left|\nabla u_{k_{j}}\right|^{m} d x\right)=0 \quad \text { (by } \quad \text { (54)). }
\end{aligned}
$$

Hence $\nabla \tilde{v} \equiv 0$ in $B_{R}(0)$, which implies $\tilde{v} \equiv 1$ in $B_{R}(0)$ since $\tilde{v}\left(0, \ldots, 0, \beta_{*}\right)=1$, and this contradicts (64).

Case 2

$P_{0} \in \Omega$. In this case we define

$$
v_{k}(z)=\tilde{M}_{k}^{-1} u_{k}\left(\lambda_{k} z+P_{k}\right)
$$

Then $v_{k}(0)=1$ for all $k=1,2, \ldots ; v_{k}$ satisfies

$$
\left\{\begin{array}{l}
\Delta_{m} v_{k}-\tilde{M}_{k}^{m-v} v_{k}^{m-1}+v_{k}^{\frac{N(m-1)+m}{N-m}}=0 \text { in } \Omega_{k}, \\
\frac{\partial v_{k}}{\partial n_{k}}=0 \quad \text { on } \partial \Omega_{k},
\end{array}\right.
$$

where $n_{k}$ is the unit outer normal of $\Omega_{k}=\left\{z \mid \lambda_{k} z+P_{k} \in \Omega\right\}$. For any $R>0$ we have $\frac{\alpha_{k}}{\lambda_{k}}>2 R$ if $k$ is sufficiently large, so that the entire ball $B_{2 R}^{-}(0)$ is contained in $\Omega_{k}$ Repeating the argument as in the subcase (i), we obtain a subsequence such that $\left\{v_{k_{j}}\right.$ is uniformly convergent to a $C^{1, \alpha^{\#}}$ function $\widehat{v}$ on any compact set of $\mathbb{R}^{N}$ and moreover it follows from (65) that $\widehat{v}$ satisfies (64) with $\bar{v}$ replaced by $\widehat{v}$. On the other hand, for any fixed $R>0$ we have

$$
\begin{aligned}
\int_{B_{R}(0)}|\nabla v|^{m} d z & =\lim _{j \rightarrow \infty} \int_{B_{R}(0)}\left|\nabla v_{k_{j}}\right|^{m} d z \leq \lim _{j \rightarrow \infty} \int_{\Omega_{k_{j}}}\left|\nabla v_{k_{j}}\right|^{m} d z \\
& =\lim _{j \rightarrow \infty} \varepsilon_{k_{j}}^{m-N} \int_{\Omega}\left|\nabla u_{k_{j}}\right|^{m} d x \\
& =\lim _{j \rightarrow \infty} \varepsilon_{k_{j}}^{-N}\left(\int_{\Omega} \varepsilon_{k_{j}}^{m}\left|\nabla u_{k_{j}}\right|^{m} d x\right)=0 \quad \text { (by } \quad \text { (54)). }
\end{aligned}
$$

Hence $\bar{v} \equiv 0$ in $B_{R}(0)$, which implies $\widehat{v} \equiv 1$ in $B_{R}(0)$ since $\widehat{v}(0)=1$, and this contradicts (64). Hence Claim 1 is proved by combining the above cases together.

\section{Claim 2}

There exists a constant $0<\mathrm{C}<\infty$ independent of $\varepsilon$ such that $\mathrm{u}_{\varepsilon} \leq \mathrm{C}$.

If we multiply both sides of (42) by $u^{m(s-1)+1_{\varepsilon}}(s \geq 1)$ and integrate over $\Omega$, then by virtue of the homogeneous Neumann boundary condition (44) it follows that

$$
s^{-m}[m(s-1)+1] \varepsilon^{m} \int_{\Omega}\left|\nabla u_{\varepsilon}^{s}\right|^{m} d x+\int_{\Omega} u_{\varepsilon}^{m s} d x=\int_{\Omega} u_{\varepsilon}^{m(s-1)+v} d x .
$$


Note that Claim 1 tells us that

$$
\int_{\Omega} u_{\varepsilon}^{m(s-1)+v} d x \leq \sup _{\Omega} u_{\varepsilon}^{\nu-m} \int_{\Omega} u_{\varepsilon}^{m s} d x \leq C \varepsilon^{m} \int_{\Omega} u_{\varepsilon}^{m s} d x
$$

Since $s^{-m}[m(s-1)+1] \leq m+1$ for $\geq 1$ we obtain from (66) and (67)that

$$
s^{-m}[m(s-1)+1] \int_{\Omega}\left(\left|\nabla u_{\varepsilon}^{s}\right|^{m}+u_{\varepsilon}^{m s}\right) d x \leq C \int_{\Omega} u_{\varepsilon}^{m s} d x
$$

Note that $\frac{s^{m}}{m(s-1)+1} \leq \frac{N}{m^{2}+(N-m)} s^{m-1}$ for $s \geq \frac{v}{m}$. Thus it follows from the Sobolev embedding theorem (19) and (68) that for $s \geq \frac{v}{m}$,

$$
\left(\int_{\Omega} u_{\varepsilon}^{v s} d x\right)^{\frac{m}{v}} \leq(C s)^{m-1} \int_{\Omega} u_{\varepsilon}^{m s} d x
$$

Next we define two sequences $\left\{\widehat{s}_{j}\right\}$ and $\left\{\widehat{M}_{j}\right\}$ by

$$
\widehat{s}_{j}=\left(\frac{v}{m}\right)^{j-1} v, \quad \widehat{M}_{j}=\int_{\Omega} u_{\varepsilon}^{\widehat{s}_{j}} d x \quad \text { for } \quad j=1,2, \ldots
$$

Note that $\widehat{s}_{j} \geq v$ for all $j \geq 0$ and $\widehat{s}_{j} \rightarrow \infty$ as $j \rightarrow \infty$. The inequality (69) yields that

$$
\left(\widehat{M}_{j+1}\right)^{\frac{1}{s_{j+1}}} \leq\left(C \widehat{S}_{j}\right)^{\frac{m-1}{m} v \cdot \frac{1}{s_{j+1}}} \widehat{M}_{j}^{\frac{1}{s_{j}}} .
$$

If we let $\widehat{\mu}_{j}=\frac{1}{\widehat{s}_{j}} \log \widehat{M}_{j}$ we get

$$
\widehat{\mu}_{j+1} \leq \widehat{\mu}_{j}+\frac{m-1}{v}\left(\frac{v}{m}\right)^{-(j-1)}\left[\log (C v)+(j-1) \log \frac{v}{m}\right]
$$

Thus we have

$$
\widehat{\mu}_{j+1} \leq \widehat{\mu}_{1}+\sum_{j=1}^{\infty} \frac{m-1}{v}\left(\frac{v}{m}\right)^{-(j-1)}\left[\log (C v)+(j-1) \log \frac{v}{m}\right]
$$

Note that

$$
\sum_{j=1}^{\infty} \frac{m-1}{v}\left(\frac{v}{m}\right)^{-(j-1)}\left[\log (C v)+(j-1) \log \frac{v}{m}\right] \leq C_{2}
$$

for some constant $C_{2}$ independent of $\varepsilon$. Therefore we get 


$$
\left\|u_{\varepsilon}\right\|_{L^{\infty}(\Omega)} \leq \lim _{j \rightarrow \infty} e^{\widehat{\mu}_{j}} \leq e^{C_{2}} e^{\widehat{\mu}_{1}}=C_{3}\left(\int_{\Omega} u_{\varepsilon}^{\nu}\right)^{\frac{1}{\nu}} .
$$

(54) tells us that

$$
\left(\int_{\Omega} u_{\varepsilon}^{v}\right)^{\frac{1}{v}} \leq|\Omega|^{\frac{1}{v}}
$$

Hence we have

$$
\left\|u_{\varepsilon}\right\|_{L^{\infty}(\Omega)} \leq C
$$

for some positive constant independent of $\varepsilon$. Proof of Theorem 2 is complete.

By applying Lemma 2 to $u_{\varepsilon}$ we have the following uniform $C^{1, \alpha}$-estimate of $u_{\varepsilon}$ as a corollary of Theorem 2.

\section{Corollary 1}

Suppose $1<\mathrm{m}<\mathrm{N}$. For $\varepsilon \in[1, \infty)$ there exist two positive constants $\alpha \in(0,1)$ and $\mathrm{C}$ depending only on $\mathrm{m}, \mathrm{N}$ and $\Omega$ such that for any least-energy solution $\mathrm{u}_{\varepsilon}$ to (42)-(44) we have

$$
\left\|u_{\varepsilon}\right\|_{C^{1, \alpha}(\Omega)} \leq C .
$$

\section{Asymptotic behaviors of least energy solutions of the critical case as $\varepsilon \rightarrow$ $\infty$}

In this section we study asymptotic behaviors of least energy solution $u_{\varepsilon}$ of the critical case as $\varepsilon \rightarrow \infty$ and give Proof of Theorem 3. We decompose $u_{\varepsilon}$ as we did before in (9), i.e., $u_{\varepsilon}$ as $u_{\varepsilon}=\bar{u}_{\varepsilon}+v_{\varepsilon}$, where

$$
\bar{u}_{\varepsilon}=\frac{1}{|\Omega|} \int_{\Omega} u_{\varepsilon} d x \quad \text { and } \quad \int_{\Omega} v_{\varepsilon} d x=0
$$

Then (42)-(44) tell us that $v_{\varepsilon}$ satisfies the following equations

$$
\left\{\begin{array}{l}
\varepsilon^{m} \Delta_{m} v_{\varepsilon}-u_{\varepsilon}^{m-1}+u_{\varepsilon}^{\frac{N(m-1)+m}{N-m}}=0 \quad \text { in } \Omega, \\
\frac{\partial v_{\varepsilon}}{\partial n}=0 \text { on } \partial \Omega .
\end{array}\right.
$$

Since

$$
u_{\varepsilon}^{m-1}-u_{\varepsilon}^{\frac{N(m-1)+m}{N-m}}-\bar{u}_{\varepsilon}^{m-1}+\bar{u}_{\varepsilon}^{-\frac{N(m-1)+m}{N-m}}=\left[(m-1)\left(\int_{0}^{1}\left(\bar{u}_{\varepsilon}+t v_{\varepsilon}\right)^{m-2} d t\right)-\frac{N(m-1)+m}{N-m} \int_{0}^{1}\left(\bar{u}_{\varepsilon}+t v_{\varepsilon}\right)^{\frac{N(m-2)+2 m}{N-m}} d t\right] v_{\varepsilon}
$$


we have

$$
\varepsilon^{m} \Delta_{m} v_{\varepsilon}-\left[(m-1) \int_{0}^{1}\left(\bar{u}_{\varepsilon}+t v_{\varepsilon}\right)^{m-2} d t-\frac{N(m-1)+m}{N-m} \int_{0}^{1}\left(\bar{u}_{\varepsilon}+t v_{\varepsilon}\right)^{\frac{N(m-2)+2 m}{N-m}} d t\right] v_{\varepsilon}=\bar{u}_{\varepsilon}^{m-1}-\bar{u}_{\varepsilon}^{-\frac{N(m-1)+m}{N-m}} .
$$

Multiplying both sides of (75) by $v_{\varepsilon}$, integrating over $\Omega$ and using integration by parts we have

$$
\varepsilon^{m} \int_{\Omega}\left|\nabla v_{\varepsilon}\right|^{m} d x+\int_{\Omega}\left[(m-1) \int_{0}^{1}\left(\bar{u}_{\varepsilon}+t v_{\varepsilon}\right)^{m-2} d t-\frac{N(m-1)+m}{N-m} \int_{0}^{1}\left(\bar{u}_{\varepsilon}+t v_{\varepsilon}\right)^{\frac{N(m-2)+2 m}{N-m}} d t\right] v_{\varepsilon}^{2} d x=0 .
$$

Theorem 2 tells us

$$
0<\bar{u}_{\varepsilon}+t v_{\varepsilon} \leq \max _{\bar{\Omega}} u_{\varepsilon}(x) \leq C .
$$

Since

$$
\lim _{t \rightarrow 0^{+}} \frac{t^{\frac{N(m-2)+2 m}{N-m}}}{t^{m-2}}=0
$$

it follows from (76) that

$$
\left|\left((m-1)\left(\bar{u}_{\varepsilon}+t v_{\varepsilon}\right)^{m-2}-\frac{N(m-1)+m}{N-m}\left(\bar{u}_{\varepsilon}+t v_{\varepsilon}\right)^{\frac{N(m-2)+2 m}{N-m}}\right)\right| \leq C
$$

for $(x, t) \in \bar{\Omega} \times[0,1]$. Thus we get by the Poincaré inequality

$$
\varepsilon^{m} \gamma^{m} \int_{\Omega}\left|v_{\varepsilon}\right|^{m} d x \leq \varepsilon^{m} \int_{\Omega}\left|\nabla v_{\varepsilon}\right|^{m} d x \leq C \int_{\Omega} v_{\varepsilon}^{2} d x .
$$

Proof of Theorem 3 Case 1. $1<m \leq 2$. It follows from (77) that

$$
\varepsilon^{m} \gamma^{m} \int_{\Omega}\left|v_{\varepsilon}\right|^{m} \leq C \int_{\Omega} v_{\varepsilon}^{2} d x \leq C \sup _{\Omega}\left|v_{\varepsilon}\right|^{2-m} \int_{\Omega}\left|v_{\varepsilon}\right|^{m} d x \leq C \int_{\Omega}\left|v_{\varepsilon}\right|^{m} d x \quad \text { (by Theorem 2). }
$$

If we take $\varepsilon>\varepsilon_{1}=\frac{\sqrt[m]{C}}{\gamma}$, we know $\int_{\Omega}\left|v_{\varepsilon}\right|^{m} d x=0$, which yields that $v_{\varepsilon} \not \equiv 0$. Hence

$$
u_{\varepsilon} \equiv \frac{1}{|\Omega|} \int_{\Omega} u_{\varepsilon} d x \quad \text { on } \quad \bar{\Omega} .
$$

Maximum Principle tells us that $\sup _{\Omega} u_{\varepsilon} \geq 1$ ([8]). Therefore $u_{\varepsilon} \not \equiv 1$ for $\varepsilon>\varepsilon_{1}$. 
Case 2. $2<m<N$. For this case, by using Hölder inequality, interpolation and the fact that $\left\|v_{\varepsilon}\right\|_{L^{\infty}(\Omega)} \leq C$ as we did in Sect. 2 we obtain that for all $q \geq m$

$$
\left\|v_{\varepsilon}\right\|_{L^{q}(\Omega)} \leq C \varepsilon^{-\frac{m^{2}}{q(m-2)}} .
$$

By repeating the proof of Lemma 1 we have

$$
\left\|v_{\varepsilon}\right\|_{L^{\infty}(\Omega)} \leq C \varepsilon^{-\beta}
$$

with $\beta$ as defined in Lemma 1. Applying Lemma 2 to $v_{\varepsilon}$ and taking care of (78) yield

$$
\left\|v_{\varepsilon}\right\|_{C^{1, \alpha}(\bar{\Omega})} \leq C \varepsilon^{-\beta}
$$

for some constants $\alpha \in(0,1)$ depending only on $m,>N$ and $\Omega$, and $C$ depending only on $m$, $N, \Omega$ and the uniform upper bound in Theorem 2. Note that $t^{m-1}-t^{\frac{N(m-1)+m}{N-m}}$ has only 1 as its positive zero. Next we show that $\bar{u}_{\varepsilon} \rightarrow 1$ as $\varepsilon \rightarrow \infty$. Since maximum principle tells us that $\sup _{\Omega} \geq 1$ ([8]) it follows from (78) and Theorem 2 that for sufficiently large $\varepsilon$,

$$
\frac{1}{2} \leq \bar{u}_{\varepsilon} \leq C
$$

To show $\bar{u}_{\varepsilon} \rightarrow 1$ as $\varepsilon \rightarrow \infty$ it is equivalent to show that for any sequence $\left\{\varepsilon_{j}\right\}$ with $\lim j \rightarrow \infty$ $\varepsilon_{j}=\infty, \lim _{j \rightarrow} \lim _{\varepsilon_{j}}=1$.

Suppose not. Then it follows from (80) that there exist a sequence $\left\{\varepsilon_{j}\right\}$ with $\lim j \rightarrow \infty \varepsilon_{j}=$

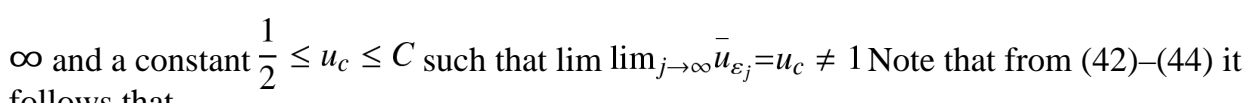
follows that

$$
\varepsilon_{j}^{m} \int_{\Omega}\left|\nabla u_{\varepsilon_{j}}\right|^{m} d x=\int_{\Omega}\left(u_{\varepsilon_{j}}^{v}-u_{\varepsilon_{j}}^{m}\right) d x .
$$

Note that (77) and (78) imply

$$
\varepsilon_{j}^{m} \int_{\Omega}\left|\nabla u_{\varepsilon_{j}}\right|^{m} d x=\varepsilon_{j}^{m} \int_{\Omega}\left|\nabla v_{\varepsilon_{j}}\right|^{m} d x \leq C \int_{\Omega} v_{\varepsilon_{j}}^{2} d x \leq C \varepsilon_{j}^{-2 \beta}|\Omega|
$$

Taking limit at both sides of (81) as $j \rightarrow \infty$ and using (79) we obtain

$$
\int_{\Omega}\left(u_{c}^{v}-u_{c}^{m}\right) d x=0
$$

which implies $u_{c}^{v}-u^{m}{ }_{c}=0$, i.e., the constant $u_{c}$ must be 1 , contradiction. Therefore we have 


$$
\left\|u_{\varepsilon}-1\right\|_{C^{1, \alpha}(\bar{\Omega})} \leq\left\|v_{\varepsilon}\right\|_{C^{1, \alpha}(\bar{\Omega})}+\left\|\bar{u}_{\varepsilon}-1\right\|_{C^{1, \alpha}(\bar{\Omega})} \rightarrow 0
$$

as $\varepsilon \rightarrow \infty$. Proof of Theorem 3 is complete.

\section{References}

1. Ambrosetti A, Rabinowitz P. Dual variational methods in critical point theory and applications. J. Funct. Anal 1973;14:349-381.

2. Díaz, J. Research Notes in Mathematics. Vol. vol. 106. Pitman Advanced Publishing Program; Boston: 1985. Nonlinear Partial Differential Equations and Free Boundaries, Vol. I: Elliptic Equations.

3. Gui C. Multipeak solutions for a semilinear Neumann problem. Duke Math. J 1996;84:739-769.

4. Gui C, Ghoussoub N. Multi-peak solutions for a semilinear N eumann problem involving the critical Sobolev exponent. Math. Z 1998;229:443-474.

5. Gui C, Wei J. Multiple interior peak solutions for some singularly perturbed Neumann problems. J. Differ. Equ 1999;158:1-27.

6. Gui C, Wei J. Multiple mixed interior and boundary peak solutions for some singularly perturbed Neumann problems. Can. J. Math 2000;52:522-538.

7. Gui C, Wei J, Winter M. Matthias Multiple boundary peak solutions for some singularly perturbed Neumann problems. Ann. Inst. H. Poincaré Anal. Non Linéaire 2000;17:47-82.

8. Li Y, Zhao C. On the structure of a class of quasilinear elliptic Neumann problems. J. Differ. Equ 2005;212:208-233.

9. Li Y, Zhao C. On the shape of least-energy solutions to a class of quasilinear elliptic Neumann problems. IMA J. Appl. Math 2007;72:113-139.

10. Li Y, Zhao C. Locating the peak of least-energy solutions to a quasilinear elliptic Neumann problems. J. Math. Anal. Appl 2007;336:1368-1383.

11. Lin C-S, Ni W-M, Takagi I. Large amplitude stationary solutions to a chemotaxis system. J. Differ. Equ 1988;72:1-27.

12. Ni W-M, Takagi I. On the Neumann problem for some semilinear elliptic equations and systems of activator-inhibitor type. Trans. Am. Math. Soc 1986;297:351-368.

13. Ni W-M, Takagi I. On the shape of least-energy solutions to a semilinear Neumann problem. Commun. Pure Appl. Math 1991;44(7):819-851.

14. Ni W-M, Takagi I. Locating the peaks of least-energy solutions to a semilinear Neumann problem. Duke Math. J 1993;70:247-281.

15. Pan XB. Singular limit of quasilinear Neumann problems. Proc. R. Soc. Edinb. Sect. A 1995;125:205-223.

16. Tolksdorf P. Regularity for a more general class of quasilinear elliptic equations. J. Differ. Equ 1984;51:126-150.

17. Wang XJ. Neumann problems of semilinear elliptic equations involving critical Sobolev exponents. J. Differ. Equ 1991;93:283-310.

18. Wang XJ. Positive solutions to the Neumann problem for $p$-Laplace equations. Gaoxiao Yingyong Shuxue Xuebao (Ser. A) 1993;8:99-112. (in Chinese).

19. Wei J. On the boundary spike layer solutions to a singularly perturbed Neumann problem. J. Differ. Equ 1997;134:104-133. 